\title{
Adding and subtracting vectors: The problem with the arrow representation
}

\author{
Andrew F. Heckler \\ Department of Physics, Ohio State University, Columbus, Ohio 43210, USA \\ Thomas M. Scaife \\ Department of Chemistry and Engineering Physics, University of Wisconsin-Platteville, \\ Platteville, Wisconsin 53818, USA \\ (Received 28 May 2014; published 20 January 2015)
}

\begin{abstract}
A small number of studies have investigated student understanding of vector addition and subtraction in generic or introductory physics contexts, but in almost all cases the questions posed were in the vector arrow representation. In a series of experiments involving over 1000 students and several semesters, we investigated student understanding of vector addition and subtraction in both the arrow and algebraic notation (using $\hat{i}, \hat{j}, \hat{k}$ ) in generic mathematical and physics contexts. First, we replicated a number of previous findings of student difficulties in the arrow format and discovered several additional difficulties, including the finding that different relative arrow orientations can prompt different solution paths and different kinds of mistakes, which suggests that students need to practice with a variety of relative orientations. Most importantly, we found that average performance in the $i j k$ format was typically excellent and often much better than performance in the arrow format in either the generic or physics contexts. Further, while we find that the arrow format tends to prompt students to a more physically intuitive solution path, we also find that, when prompted, student solutions in the $i j k$ format also display significant physical insights into the problem. We also find a hierarchy in correct answering between the two formats, with correct answering in the $i j k$ format being more fundamental than for the arrow format. Overall, the results suggest that many student difficulties with these simple vector problems lie with the arrow representation itself. For instruction, these results imply that introducing the $i j k$ notation (or some equivalent) with the arrow notation concurrently may be a very useful way to improve student performance as well as help students to learn physics concepts involving vector addition and subtraction.
\end{abstract}

DOI: $10.1103 /$ PhysRevSTPER.11.010101

PACS numbers: 01.40.Fk, 01.40.Ha, 01.40.gb

\section{INTRODUCTION}

Vector addition and subtraction are fairly common in the introductory physics curriculum, and a number of researchers have documented a range of student difficulties with these operations in generic mathematical contexts as well as in physical contexts [1-7]. However, in virtually all of these studies, only the arrow representation of vectors was considered. While the arrow representation is undoubtedly pedagogically, intuitively, conceptually, and technically useful, it also carries with it significant student preconceptions, and it is limited in its usefulness in calculations, in its ability to characterize some important properties of vectors, and in its ability to represent vector quantities (for example, in three or more dimensions). One could compare the arrow representation and its attendant advantages and disadvantages with, for example, the algebraic representation using unit vectors $(\hat{i}, \hat{j}, \hat{k})$, which is sometimes used in

Published by the American Physical Society under the terms of the Creative Commons Attribution 3.0 License. Further distribution of this work must maintain attribution to the author(s) and the published article's title, journal citation, and DOI. instruction, particularly in the calculus-level physics courses. Are there critical and more comprehensive aspects of vectors and vector operations that are not well represented, or even inhibited, by the arrow representation of vectors? Does the $i j k$ format complement the arrow format or further inhibit student understanding and performance? In this study, we would like to gain deeper insights into student understanding of critical aspects of vectors and vector addition and subtraction in a generic math format as well as in simple physics contexts by investigating student responses to questions in both the arrow and $i j k$ formats. Not only will this provide interesting information about student understanding of a critical topic, but it may also be used to help improve instructional practices and student understanding.

As mentioned, there are a number of studies investigating student understanding only in the context of the arrow format. For example, Nguyen and Meltzer [1] found that only about $60 \%-70 \%$ of calculus-based students and $20 \%-40 \%$ of algebra-based students can correctly draw the sum of two vectors represented as arrows on a two-dimensional grid, and only $60 \%-80 \%$ of all students could correctly answer basic magnitude and direction comparison questions about arrows on a grid. Flores, 
Kanim, and Kautz [2] represented vectors as arrows with given lengths and relevant angles (rather than arrows on a grid), and found that in a physics context of summing vectors to find the net force, only about $50 \%$ of calculusbased students could answer the questions correctly. They also found that significantly fewer students could answer qualitative questions involving the subtraction of vectors in a physics context (acceleration) in which the vectors were arrows drawn on a picture (with no grid). Shaffer and McDermott [3] also found that many students had difficulty with vector subtraction in which vectors were represented as arrows on a one-dimensional grid, and students did marginally better ( $65 \%$ correct) in the generic math context compared to the physics context of acceleration (45\% correct). In contrast, Van Deventer and Wittmann [4] found that students did better in the physics (50\% correct) compared to the math context (20\% correct) for onedimensional subtraction, because students tended to use the physical context to obtain the correct signs. Barniol and Zavala [5] have also found that student responses to test questions in which they must draw the resultant vectors as arrows on a grid can be somewhat sensitive to the context of the question. Student responses to vector addition questions can also be somewhat sensitive to the placement of the vectors represented as arrows on the page, such as tail to tail, tail to tip, or separated $[5,6]$, though no effects were found from placing the arrows on a grid [6].

While none of the above studies reported using the $i j k$ format, we found two studies that did. The first study involved the development and validation of an assessment instrument by Barniol and Zavala [7], which assesses a number of basic vector skills. However, most of the items were in the arrow format; the few questions in $i j k$ format were for vector products, calculation of magnitudes and directions, and one item in which $92 \%$ of students successfully translated between $i j k$ format and arrow format. All items on addition and subtraction were only in the arrow format, with scores similar to the studies described above.

The second study by Knight [8] included a mix of generic arrow and $i j k$-format questions. The results are somewhat ambiguous because many students left many of the questions blank, and about half of the students indicated that they were not familiar with the $i j k$ notation. Nonetheless, for a simple vector addition question, about $52 \%$ of students answered correctly in the $i j k$ format and only about $43 \%$ answered correctly in the arrow format.

With a more careful design, this study will demonstrate an even more dramatic difference, namely, that significantly, and often overwhelmingly, more students can (post traditional instruction) correctly add and subtract vectors in the $i j k$ format compared to the arrow format in both generic and physics contexts. While on some level this difference in performance may not come as a surprise to instructors, it does bring up several important questions: Do students answering correctly in the $i j k$ format still "not really understand" vector addition and subtraction? What do we want to mean when we want students to "understand" vectors and vector addition and subtraction? Is the poor performance in the arrow format an indication of poor understanding of the underlying physics or is it an artifact of the representation that hinders correct application of vector concepts and operations in a generic and physical context? We typically teach arrow before $i j k$ format-but is this appropriate? Is there a more natural hierarchy; that is, would it be better to introduce $i j k$ format first? Should an explicit instructional goal be for students to successfully translate or explain vector addition and subtraction in both formats, or is one format sufficient?

While some of the questions above clearly require focused empirical investigations, other questions that require more professional judgment and community discussion can also be better informed with such investigations. Therefore, in order to gain insight into answers to all of these questions, we begin by reporting on a series of experiments designed to empirically document and better characterize student performance on and understanding of simple vector addition and subtraction problems in generic and physics contexts in both the arrow format and the $i j k$ format.

\section{PARTICIPANTS AND METHOD}

The participants in this study were students enrolled in a first semester (Mechanics) or second semester (Electricity and Magnetism) introductory physics course. The participants were enrolled in the calculus-based versions of these courses, primarily designed for engineering students. Each semester, we enrolled 2-6 lecture sections (typically all sections for a particular course). Each lecture section had a typical enrollment of 200, and the courses are taught via traditional methods, with 2-3 lectures per week, 1 recitation per week, and 1 lab per week. We were not instructors for any of the sections during this study.

For data collection, students were given a "flexible homework" assignment as part of their regular course credit. They received full credit for participation. The flexible homework assignment consisted of participating in a 1-hour session in our physics education research laboratory, where students completed some combination of training, testing, and interviewing of various physics related tasks, including the tasks reported here. Data reported in this study are from these sessions. Each week during the course we would randomly select students to sign up for an appointment to complete the assigned flexible homework. All students are assigned the flexible homework and given ample opportunity to participate in one of the many scheduled sessions throughout the semester (or they can opt to do a take-home assignment); typically, over $95 \%$ participated, and the remainder of the students, for unknown reasons, did not complete the 


\begin{tabular}{|c|c|c|c|}
\hline Question type & Arrow format & \multicolumn{2}{|c|}{$i j k$ format } \\
\hline \multirow{3}{*}{$\begin{array}{c}\boldsymbol{p p p} \\
\text { (positive plus } \\
\text { positive) }\end{array}$} & What is $\vec{A}+\vec{B}(\vec{A}$ plus $\vec{B}) ?$ & \multicolumn{2}{|c|}{ What is $\vec{A}+\vec{B}(\vec{A}$ plus $\vec{B}) ?$} \\
\hline & $\square|\longmapsto| \| \square$ & $\vec{A}=5 \hat{\imath}$ & $\vec{B}=3 \hat{\imath}$ \\
\hline & $\vec{A}$ & & \\
\hline \multirow{3}{*}{$\begin{array}{c}\text { ppn } \\
\text { (positive plus } \\
\text { negative) }\end{array}$} & What is $\vec{A}+\vec{B}(\vec{A}$ plus $\vec{B}) ?$ & \multicolumn{2}{|c|}{ What is $\vec{A}+\vec{B}(\vec{A}$ plus $\vec{B}) ?$} \\
\hline & $\square \longrightarrow$ & $\vec{A}=6 \hat{\imath}$ & $\vec{B}=-2 \hat{\imath}$ \\
\hline & $\vec{A}$ & & \\
\hline \multirow{3}{*}{$\begin{array}{c}\boldsymbol{p m p} \\
\text { (positive minus } \\
\text { positive) }\end{array}$} & What is $\vec{A}-\vec{B}(\vec{A}$ minus $\vec{B})$ ? & \multicolumn{2}{|c|}{ What is $\vec{A}-\vec{B}(\vec{A}$ minus $\vec{B})$ ? } \\
\hline & \begin{tabular}{|l|l|l|l|l|l|} 
& & & & $\rightarrow$ & $\rightarrow$ \\
\end{tabular} & $\vec{A}=1 \hat{\imath}$ & $\vec{B}=4 \hat{\imath}$ \\
\hline & $\vec{A}$ & & \\
\hline \multirow{3}{*}{$\begin{array}{c}\text { pmn } \\
\text { (positive minus } \\
\text { negative) }\end{array}$} & What is $\vec{A}-\vec{B}(\vec{A}$ minus $\vec{B})$ ? & \multicolumn{2}{|c|}{ What is $\vec{A}-\vec{B}(\vec{A}$ minus $\vec{B})$ ? } \\
\hline & $\square \longrightarrow$ & $\vec{A}=5 \hat{\imath}$ & $\vec{B}=-3 \hat{\imath}$ \\
\hline & $\vec{A}$ & & \\
\hline \multirow{3}{*}{$\begin{array}{l}\text { Response choices } \\
\text { for pmn question } \\
\text { above. }\end{array}$} & $\vec{A}-\vec{B}=\quad($ choose one $)$ & \multirow{2}{*}{$\begin{array}{r}\vec{A}-\vec{B}= \\
\text { a. }-8 \hat{\imath}\end{array}$} & (choose one) \\
\hline & $\square \underset{\text { a. }}{\longrightarrow} \underset{\text { b. }}{\longrightarrow}$ & & b. $8 \hat{\imath}$ \\
\hline & $\underset{\text { c. }}{\square+\mid}$ & c. $-2 \hat{\imath}$ & d. $2 \hat{\imath}$ \\
\hline
\end{tabular}

FIG. 1. Examples of one-dimensional questions in arrow and $i j k$ format.

assignment. During the flexible homework session, students were asked to answer the questions as best they could even if they have not seen the material yet. They sat at individual stations in a quiet, proctored room to answer several series of physics questions either with pencil and paper or on the computer. Students completed the material at their own pace. Afterward we would informally ask students whether they had any questions and/or to explain their answers. We observed during these sessions that almost all students made an earnest effort to answer the questions, and the complete and often detailed responses to the questions also supported this observation. Finally, note that all items were administered at least several weeks after the relevant instruction and homework, and we found no significant temporal variations in performance over period of the data collection.

\section{VECTOR ADDITION AND SUBTRACTION IN ONE DIMENSION}

In order to obtain a more systematic understanding of student difficulties with vector addition and subtraction, we began with questions in one dimension and included combinations of vectors pointing in the same direction and in opposite directions. This resulted in four kinds of questions: $p p p$ (vector in positive direction plus a vector in positive direction), $p p n$ (positive plus negative), $p m p$ (positive minus positive), and pmn (positive minus negative). Examples are shown in arrow and $i j k$ format in
Fig. 1. Note that we reiterated the question in word-andsymbol format (e.g., " $A$ minus $B$ ") in order to better ensure that the participants were not simply misreading the operation and inadvertently mistaking addition for subtraction. Post interviews confirmed that they understood the questions.

First, in a series of pilot tests, we posed the questions in free-response format with an empty grid for drawing arrows or blank spaces for writing the $i j k$ format. In this way we determined that the most typical errors were equivalent to adding instead of subtracting or an overall sign error. This provided four answer choices for all tasks (adding or subtracting the two vectors and the resultant vector in positive or negative direction), and we used these choices to construct multiple-choice questions: one choice was correct and the others were common incorrect answers (see Fig. 1).

The pilot tests indicated that most students were at ceiling for the $i j k$ format, but not for the arrow format. Therefore, when we ran the main experiment, we placed most students in the arrow-format condition and (randomly) assigned 241 students to the arrow-format condition and 37 students to the $i j k$-format condition. We presented three questions in a row for each type of question ( $p p p, p n p$, etc.), randomly varying the lengths of the vectors to provide variety. In order to reduce the effects of question order, we counterbalanced each condition, with half of the participants answering the questions in reverse order.

The results from first-semester calculus-based physics students are presented in Fig. 2. Participants in the $i j k$ 


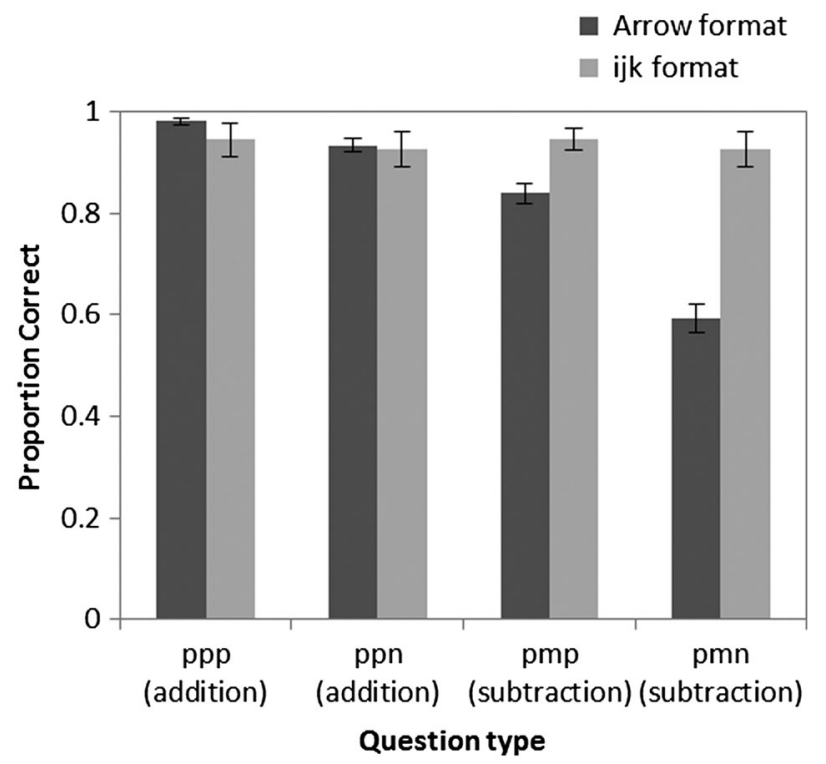

FIG. 2. Student performance on one-dimensional vector questions (see Fig. 1). Scores were high for all question types in the $i j k$ format. Scores were lower for subtraction questions, especially for subtraction of opposing vectors $(\mathrm{pmn})$. Error bars indicate \pm 1 standard error (SE).

condition scored very high, with means greater than $93 \%$ for all addition and subtraction question types. However, in the arrow condition, the mean scores were high for the addition questions $(>93 \%)$, but lower for the subtraction questions. Specifically, for the $p m p$ questions when the arrows were in the same direction, scores were only slightly lower (84\%), but for the pmn questions when the arrows opposed each other, the scores were dramatically lower (59\%). Note that we found that arrows pointing inward versus pointing outward did not significantly change the student responses. An examination of the distribution of scores revealed that in the arrow condition about 32\% of participants answered incorrectly on all three pmn questions.

A closer examination of the subtraction responses revealed that most errors were the result of effectively adding the two vectors instead of subtracting them. We interviewed 10 additional students while doing this task, and there was a tendency to think that the opposing arrows were acting against each other and the goal was to determine the result of this "competition" by finding which one "wins" and by how much, which is effectively the same as adding the two vectors. For some students, it was also clear that for $p m n$ questions (only) they were confusing the negative sign of the opposing vector with the task of subtracting the vectors, and were not realizing that both issues (the negative sign and the subtraction) had to be accounted for.

In summary, for one-dimensional vectors, we find that students were highly proficient at adding vectors in either the arrow or $i j k$ format, but for subtraction, many students answered incorrectly in the arrow format when the arrows were in opposite directions, but not when they were in the same direction - in some sense many students appeared to think that the opposition of the arrows accounted for the subtraction, and they effectively added instead of subtracted. These issues did not appear for the $i j k$ format, in which students had high proficiency in the vector subtraction tasks. Note that Shaffer and McDermott [3] found scores similar to ours for subtraction of opposing vectors in the arrow format; our results with other orientations and addition indicate that the difficulties are the most significant for this orientation and format.

\section{VECTOR ADDITION AND SUBTRACTION IN TWO DIMENSIONS}

In this section, we first compare student performance on generic two-dimensional vector addition and subtraction in the arrow and $i j k$ formats, and follow up with a more detailed investigation of performance in the arrow format, where students have the most difficulties.

\section{A. ijk format versus arrow format}

Midway through a calculus-based semester 1 physics course, 203 students were randomly assigned to one of four groups receiving either two-dimensional arrow or $i j k$ format questions and either addition or subtraction questions. The questions were in a simple generic mathematical context. An example of the questions is shown in Fig. 3. As might be expected from the one-dimensional results above, a two-way analysis of variance (ANOVA) (vector operation $\times$ format) revealed that both main effects were significant, and there was no significant interaction between the factors. Specifically, scores were significantly higher in the $i j k$ format compared to the arrow format $\left[F(1)=31.2, p<0.0001, \eta^{2}=0.13\right]$. The scores for the $i j k$ format versus arrow format for subtraction were $88 \%$ versus $52 \%(d=0.9)$ and for addition were $96 \%$ versus $72 \%(d=0.7)$, respectively. These numbers also clearly indicate that scores for addition were higher than subtraction in both formats in two dimensions $[F(1)=6.3$, $\left.p=0.01, \eta^{2}=0.03\right]$. Note that (once again) the most common error for the small number of students who incorrectly answered the subtraction question in the $i j k$ format was adding the vectors (in both dimensions) instead of subtracting them.

\section{B. Arrow format: Addition versus subtraction}

In order to obtain a more detailed understanding of student difficulties with the arrow format, we posed a variety of two-dimensional arrow-format addition and subtraction questions to 186 students in semester 1 physics and a separate population of 94 students in semester 2 physics, both calculus based. Since we found earlier that students had difficulty subtracting opposing vectors in the 
Consider the vectors below:
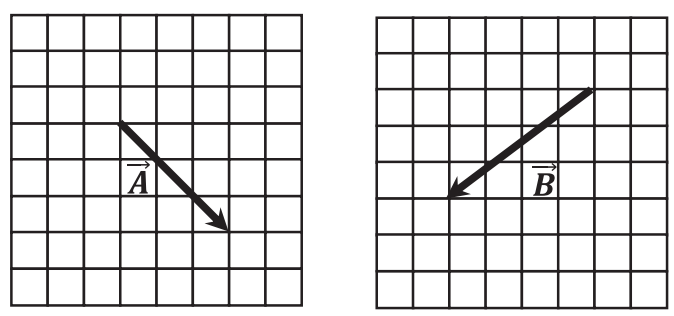

What is $\overrightarrow{\boldsymbol{A}}-\overrightarrow{\boldsymbol{B}}(\vec{A}$ minus $\vec{B})$ ? Draw your answer on the grid below:

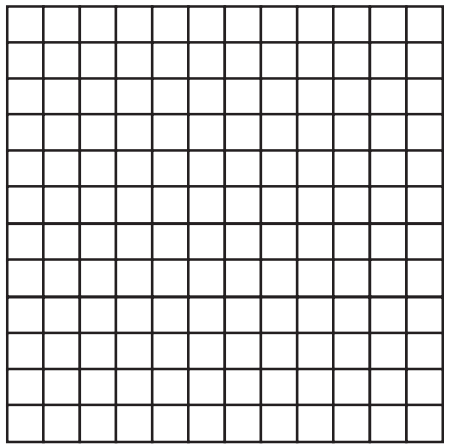

Consider the vectors below:
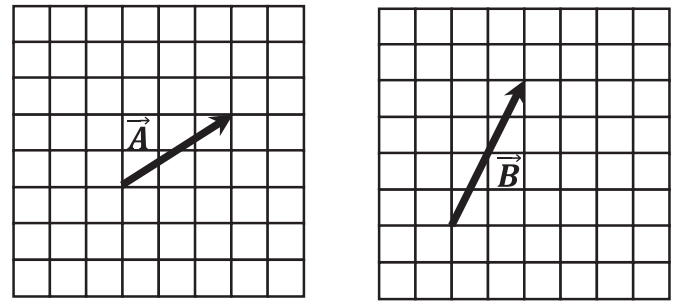

What is $\overrightarrow{\boldsymbol{A}}-\overrightarrow{\boldsymbol{B}}$ ( $\vec{A}$ minus $\vec{B})$ ?

Draw your answer on the grid below:



Consider the vectors below:

$$
\vec{A}=3 \hat{\imath}+3 \hat{\jmath} \quad \vec{B}=-4 \hat{\imath}+3 \hat{\jmath}
$$

What is $\overrightarrow{\boldsymbol{A}}-\overrightarrow{\boldsymbol{B}}(\vec{A}$ minus $\vec{B})$ ?

FIG. 3. Examples of two-dimensional subtraction questions in arrow and $i j k$ format.

arrow format in one dimension, we investigated cases in which the vectors were opposed in one of the dimensions but aligned (i.e., in the same direction) in the other, and also cases in which the vectors were aligned in both dimensions. Figure 3 presents examples of questions posed, and Figs. 4 and 5 present some of the results. The results are summarized as follows.

\section{Scores for addition are higher than for subtraction}

As indicated in Fig. 4, while the total scores on the vector addition condition (79\%) are not as high as one would hope for a relatively simple task, the performance is significantly higher than for vector subtraction condition (57\%) $[t(185)=3.15, p=0.002, d=0.5]$. Note that Ngyuen and Meltzer [1] posed a question related to subtraction in the arrow format (i.e., find the missing addend), and our subtraction scores are similar to those results, but our mean scores for addition are somewhat higher. Here, we compare performance on explicit addition and subtraction tasks.

\section{Subtraction error types depend on relative orientation of arrow components}

Figure 4 presents results for the case when the vectors are opposing in one dimension but not the other (see example

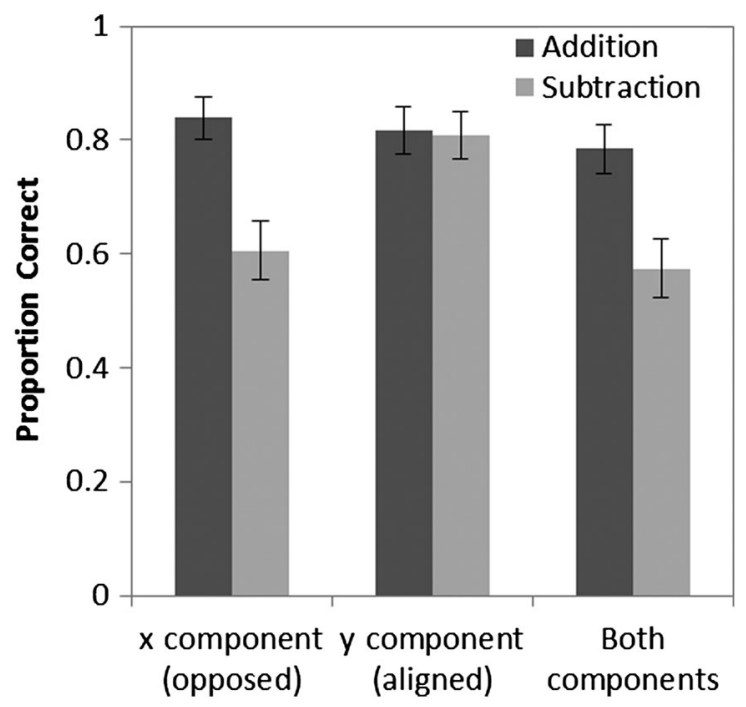

FIG. 4. Student performance ( $N=187$, semester 1$)$ on addition and subtraction of two vectors in the arrow format for questions in which the $x$ components were in opposing directions and the $y$ components were in the same direction (see Fig. 3, left-hand side). The categories represent proportion of students correctly adding (or subtracting) the $x$ components, which are in opposing directions, the $y$ components, which are pointed in the same direction, and the combined total scores of both components. Error bars indicate \pm 1 SE. 


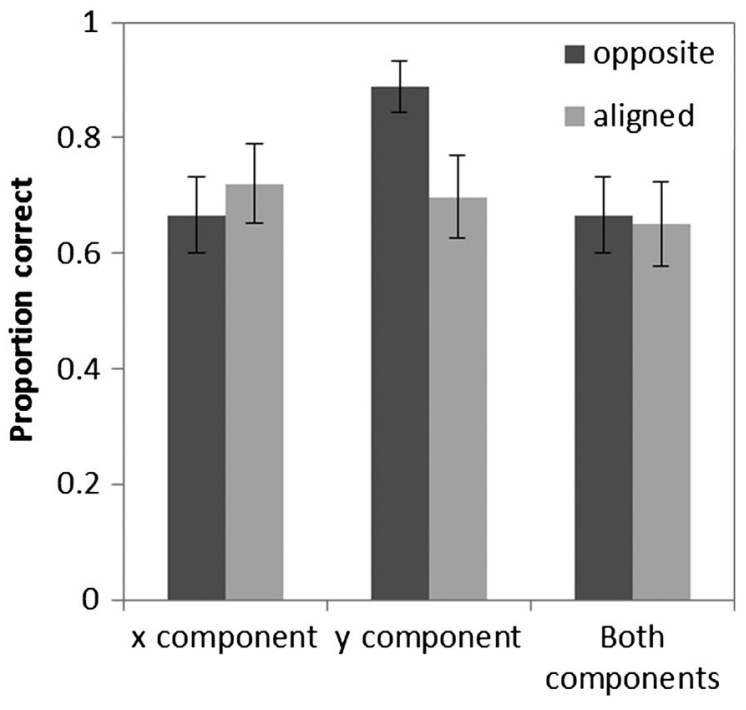

FIG. 5. Student performance ( $N=94$, semester 2$)$ on subtraction of two vectors in the arrow format for cases in which either components in both dimensions were in the same direction (aligned) or components in one dimension were aligned and components were opposed in the other dimension (see Fig. 3). For the latter case, the $x$ components were in opposing directions and the $y$ components were in the same direction. The categories represent the proportion of students correctly subtracting the $x$ components (which were opposed or aligned), the $y$ components (which were aligned), and the combined total scores of both components. Error bars indicate $\pm 1 \mathrm{SE}$.

in the left-hand side of Fig. 3); interestingly, and perhaps as one would seem to expect from the our one-dimensional results, students fairly successfully subtracted the dimension in which the vectors were aligned $(81 \%)$, but were significantly less successful for the dimension in which the vectors were opposed $(61 \%)$ [paired $t(93)=4.1$, $p<0.0001, d=0.45]$. In contrast, in the case of addition, Fig. 4 shows no difference in the performance for the dimension in which the vectors were opposed versus when they were aligned.

While the results that subtraction in the opposing dimension is worse than for the aligned dimension are consistent with the one-dimensional results, we found that the story is more complicated than the explanation that students simply do worse when subtracting opposing dimensions. If this explanation were true, then from the one-dimensional results, one would expect that performance would be comparatively high for subtraction of two vectors that were aligned in both dimensions, such as in the example shown in the right-hand side of Fig. 3. However, we found this not to be the case.

Figure 5 presents the results for aligned versus opposing $2 \mathrm{D}$ vector subtraction from second-semester calculus-based physics students. We replicated the finding that for the condition with one opposing $(x)$ and one aligned $(y)$ dimension the performance for the opposing dimension was lower than for the aligned dimension (though the overall scores were expectedly higher for this secondsemester population). However, surprisingly, the performance in both dimensions for the aligned-vector condition was just as low as for the opposing dimension in the opposing vector condition. This resulted in an overall similar score for subtraction of opposing or aligned 2D vectors.

A closer analysis of the errors indicated that students tended to commit different kinds of errors for the two different relative vector orientations. For example, while about $11 \%$ of students subtracted in one dimension and added in the other for the question that had arrows with only one opposing dimension, none of the students made this error when both dimensions were aligned. In this case, they tended to make other errors, such as incorrectly finding the negative of a vector, or placing $\vec{A}$ and $-\vec{B}$ tail to tail and incorrectly summing them.

These results suggest that about $30 \%-40 \%$ of this population did not know how to subtract two-dimensional vectors in the arrow format, and different relative orientations cue different error paths. Furthermore, while the errors for the one-dimensional results are also present in two dimensions, there are qualitatively different errors that occur in two dimensions

\section{No difference in opposing arrows pointing away or toward each other}

In the example shown on the upper left-hand side of Fig. 3, the two arrows point toward each other (in one of the dimensions). We also included examples in which the arrows pointed away from each other in one of the dimensions in order to see if students would answer these two kinds of questions differently. We found no statistically significant differences in the errors or error rates between questions with the arrows "pointing toward" or "pointing away" from each other. As noted earlier, we also found this to be true in one-dimensional questions.

\section{There are several types of common errors for subtraction in arrow format}

Others have described common errors for vector addition (such as putting the arrows tail to tail and finding a kind of average direction, or completing a triangle), but students in our population tended to do fairly well with addition tasks, and had other kinds of errors when subtracting vectors. The most common errors were as follows.

(a) Overall sign error. About 10\%-20\% of students responded with an overall sign error. Responses and follow-up interviews indicated that this appeared to result from either a confusion about or lack of attention to which sense the arrow should point.

(b) Add instead of subtract. About 10\%-15\% of students added the vectors (in both dimensions), even in a task in which they are asked to subtract. In interviews, we 
found that some of these errors were simple oversights and were corrected by simply asking students to explain their answer and they would realize their error.

(c) Add one dimension, subtract in the other. When one of the dimensions has opposing components, about $5 \%-10 \%$ of students added (instead of subtracted) the vectors in the opposing dimension and correctly subtracted in the dimension in which the components were aligned. This is consistent with our onedimensional findings in which students incorrectly subtracted when the arrows were opposed.

(d) Incorrectly determine the negative of a vector. Many students explicitly realized that $\overrightarrow{\boldsymbol{A}}-\overrightarrow{\boldsymbol{B}}=\overrightarrow{\boldsymbol{A}}+(-\overrightarrow{\boldsymbol{B}})$, but about $5 \%$ of students explicitly incorrectly determined (drew) $-\overrightarrow{\boldsymbol{B}}$ by negating only one of the components rather than both.

\section{VECTOR ADDITION AND SUBTRACTION IN A PHYSICS CONTEXT}

Several authors (see Refs. [2,3]) posed vector questions in the arrow format requiring vector arithmetic in the context of physics questions, rather than in a generic mathematical context, and noted that students had significant difficulties. Here we would like to determine whether students are able to successfully answer such questions in the $i j k$ format, thus providing insight into whether the student difficulties may be with answering the physics question within a particular representation.

We posed a two-dimensional addition and a onedimensional subtraction question in a physics context to 130 semester 1 students randomly assigned to the arrow or $i j k$-format conditions, shown in Figs. 6 and 7. The twodimensional question is similar to a sum-of-forces question investigated by Flores, Kanim and Kautz [2], and the onedimensional question is similar to the acceleration question investigated by McDermott and Shaffer [3]. Overall performance in the $i j k$ format was better, and sometimes dramatically better, than in the arrow format, as shown in Fig. 8 and described in more detail below.

\section{A. Two-dimensional net force question}

For the two-dimensional addition question, students performed quite well in the $i j k$ format $(91 \%)$ and poorly in the arrow format $(32 \%)[t(128)=8.6, p<0.0001$, $d=1.5]$. Some of the errors were similar to the errors in the generic vector tasks described above, but many of the errors were different. For example, for the two-dimensional net force comparison question (Fig. 6), 33\% of students in the arrow condition simply added the magnitudes of all of the vectors regardless of direction, $10 \%$ incorrectly argued that the net force is zero in both cases because the "forces cancel," and about 20\% had miscellaneous incorrect reasoning, such as an incorrect application of the Pythagorean theorem to find a net force.
In the $i j k$ format for the two-dimensional question, almost all of the students correctly summed the vectors and provided the correct answer with correct reasoning. Very few students made errors, but about $5 \%$ found the magnitudes of the vectors then summed the magnitudes to find an (incorrect) answer.

\section{B. One-dimensional acceleration question}

For the one-dimensional subtraction question, students did fairly well in the $i j k$ format (80\% with correct answer and justification) and worse in the arrow format (64\%) $[t(128)=2.05, p=0.04, d=0.3]$. Consistent with our earlier findings, a common mistake in the arrow format was adding the vectors instead of subtracting them $(15 \%$ of students), whereas only about $7 \%$ of students in the $i j k$ format made this mistake.

\section{HIERARCHY IN ARROW- VERSUS ijk-FORMAT PERFORMANCE}

The higher performance in the $i j k$ format prompts a number of questions. First, can the student success in the $i j k$ format somehow be used to improve the performance in the arrow format? A full instructional intervention is beyond the scope of this study; instead, we prompt (or prime) students on thinking about the $i j k$ format by first giving them an explicit $i j k$-format question followed by an arrowformat question and compare this performance to students who have not been primed with the $i j k$-format question. Second, is there a hierarchy in performance between the arrow and $i j k$ formats? A hierarchy would imply that performing well in one format implies performing well in the other, but not vice versa, and this can be determined by examining within-student data for both question formats.

We collected such data to help provide answers to these questions, but first we note that we also address another issue: the results we have obtained so far show that students often perform poorly using an arrow format on a coordinate grid. However, the arrow format is commonly presented with angles relative to a coordinate system rather than on a grid. Thus, in this study we will also consider student performance in the arrow-angle format in addition to the arrow-grid and $i j k$ format.

We randomly assigned 221 students to one of four conditions in which two vector addition questions were posed in a specific combination of question formats: (1) arrow grid, $i j k$; (2) $i j k$, arrow grid; (3) arrow angle, $i j k$; (4) $i j k$, arrow angle. See Fig. 6 for examples. In addition, we also collected the final course grades for each student. The following subsections describe three analyses of these data.

\section{A. Comparison of performance on grid versus angle versus $i j k$ format}

A one-way ANOVA of the scores on the first question answered in each condition reveals a difference in scores 


\section{Arrow-grid format}

Shown below are force diagrams for two different objects, C and D.
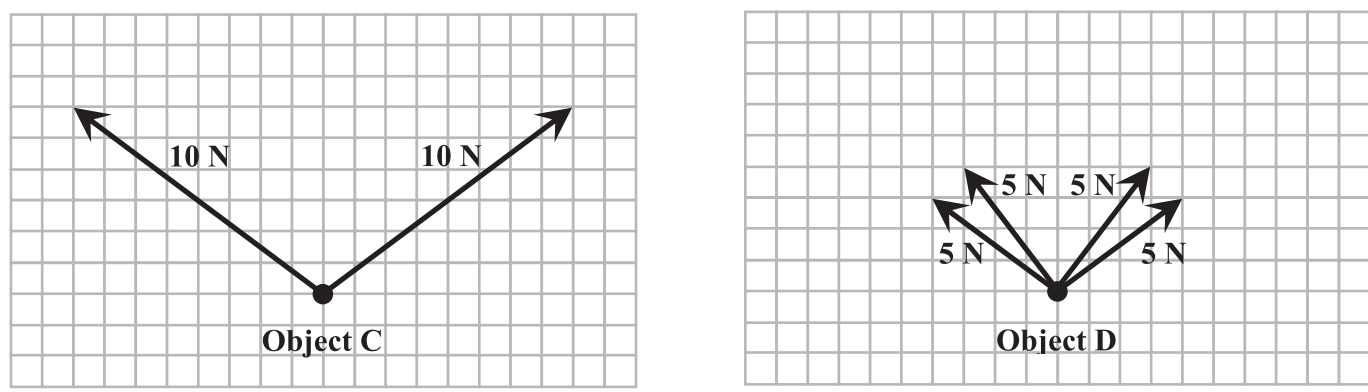

Is the magnitude of the net force on object $\mathrm{C}$ greater than, less than, or equal to the magnitude of the net force on object D? Explain or show how you determined your answer.

\section{ijk format}

Listed below are the forces exerted on two different objects, $\mathrm{C}$ and $\mathrm{D}$.

Forces on object $\mathbf{C}$

(in units of Newtons):

$$
\begin{array}{ll} 
& \vec{F}_{D 1}=-4 \hat{\imath}+3 \hat{\jmath} \\
\vec{F}_{C 1}=-8 \hat{\imath}+6 \hat{\jmath} & \vec{F}_{D 2}=+4 \hat{\imath}+3 \hat{\jmath} \\
\vec{F}_{C 2}=+8 \hat{\imath}+6 \hat{\jmath} & \vec{F}_{D 3}=-3 \hat{\imath}+4 \hat{\jmath} \\
& \vec{F}_{D 4}=+3+4 \hat{\jmath}
\end{array}
$$

\section{Forces on object D}

(in units of Newtons):

Is the magnitude of the net force on object $\mathrm{C}$ greater than, less than, or equal to the magnitude of the net force on object D? Explain or show how you determined your answer.

\section{Arrow-angle format}

Listed below are the forces exerted on two different objects, C and D.



Object C

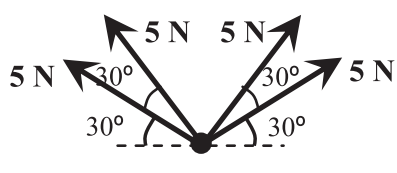

Object D

Is the magnitude of the net force on object $\mathrm{C}$ greater than, less than, or equal to the magnitude of the net force on object D? Explain or show how you determined your answer.

FIG. 6. Examples of two-dimensional physics context questions in arrow grid, arrow angle, and $i j k$ formats.

between formats $[F(2)=17, p<0.001]$, and a Tukey test shows that the mean score for the $i j k$ format $(86 \%)$ was significantly greater than either the arrow-angle score $(61 \%)$ or the arrow-grid score $(47 \%)(p<0.001)$.
Furthermore, the performance for each format for students in the lower and upper half of course grades is presented in Fig. 9. Clearly, almost all students regardless of grade can complete the $i j k$-format task, but there is a large 


\section{Arrow-grid format}

Over the same time interval, Mass A and Mass B have the initial and final velocities indicated below:

Mass A

initial velocity

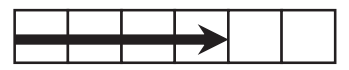

initial velocity

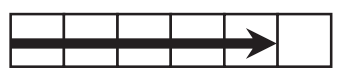

final velocity

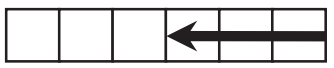

final velocity

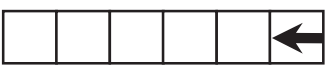

Is the magnitude of the acceleration of Mass A greater than, less than, or equal to the magnitude of acceleration of Mass B? Briefly explain and/or show your work.

\section{ijk format}

Over the same time interval, Mass A and Mass B have the initial velocities $\vec{v}_{i}$ and final velocities $\vec{v}_{f}$ indicated below (units are in $\mathrm{m} / \mathrm{s}$ ):

Mass A

$$
\begin{array}{lll}
\vec{v}_{i A}=4 \hat{\imath} & \vec{v}_{f A}=-3 \hat{\imath} \\
\vec{v}_{i B}=5 \hat{\imath} & & \text { Mass B } \\
& & \vec{v}_{f B}=-1 \hat{\imath}
\end{array}
$$

Is the magnitude of the acceleration of Mass A greater than, less than, or equal to the magnitude of acceleration of Mass B? Briefly explain and/or show your work.

FIG. 7. Examples of one-dimensional physics context questions in arrow and $i j k$ formats.



FIG. 8. Student performance $(N=130)$ on two-dimensional (Fig. 6) and one-dimensional (Fig. 7) physics context questions in both the arrow (grid) and $i j k$ formats. Error bars indicate $\pm 1 \mathrm{SE}$. discrimination between high and low grade students on the both the grid and angle-arrow format tasks. Note also that the arrow-angle format tends to solicit better performance than the arrow-grid format. Discussion of this is beyond the scope of this paper, but we noticed that this may be due to the fact that arrow-angle format tends to cue more formal ways of thinking about the component of the vectors, whereas the grid format tends to cue less formal, incorrect reasoning, but both formats often tend to cue students to think mainly about magnitudes of the full vector.

\section{B. Effect of priming with the $i j k$ format}

Figure 10 presents the score on both arrow-format questions either with or without " $i j k$ priming" (i.e., presenting a similar $i j k$-format question first), separated out by low and high grade students. This figure reveals an interesting interaction between grade and the effect of priming with the $i j k$ format, namely that for the low grade students a two-way ANOVA reveals that students score significantly higher when they are primed with the ijk format $(61 \%)$ compared to no priming $(39 \%)[F(1)=6.7$, $p=0.01, d=0.45]$ and that students score marginally 




FIG. 9. Student performance $(N=221)$ on two-dimensional physics context question (Fig. 6) separated by student grades above or below the median course grade (high grade or low grade). Error bars indicate $\pm 1 \mathrm{SE}$.

significantly higher on angle (57\%) versus grid (43\%) $[F(1)=2.94, p=0.09, d=0.3]$. However, for high grade students, there is no significant difference in score between grid and angle formats or between $i j k$ priming and no priming, perhaps because the scores are fairly high $(70 \%-80 \%)$,

This increase in performance with $i j k$-format priming (for low grade students) would suggest that while many students may fail in the arrow representation, many may nonetheless be able to correctly solve the arrow-format questions if put in the proper context, and putting students

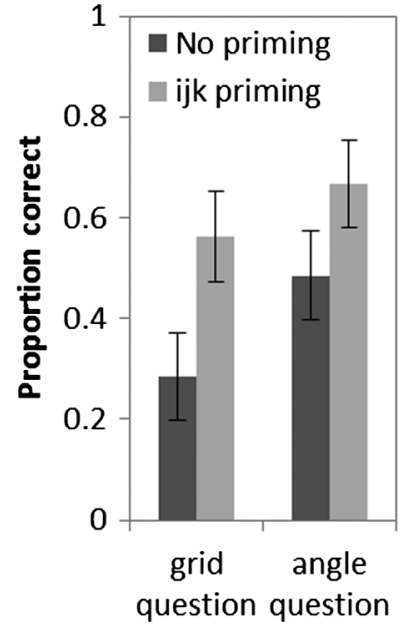

Low Grade students

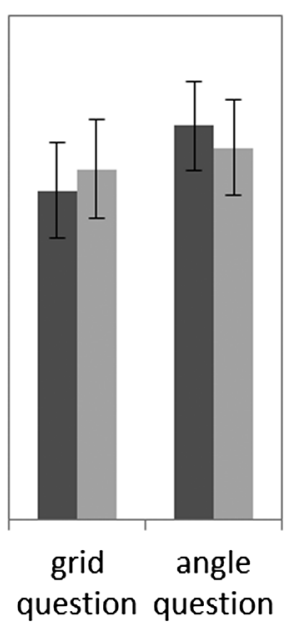

High Grade Students
FIG. 10. Student performance $(N=221)$ on arrow-angle and arrow-grid formats (Fig. 6) with and without first answering a similar $i j k$-format question (" $i j k$ priming") for low course grade and high course grade students. Error bars indicate \pm 1 SE. in the mindset of the $i j k$ format (here, via priming), which virtually all students are successful at, is one way to help them successfully complete the arrow-format task.

Note that priming with the arrow format had no such effect. That is, priming with the arrow format first had no significant effect on $i j k$-format performance, though perhaps because the scores on the $i j k$-format are near ceiling.

\section{Hierarchy of performance}

A hierarchy of performance between the arrow and $i j k$ format would imply that answering correctly in one format would strongly predict answering correctly in the other, but not vice versa (see, e.g., Table VIII in Ref. [9] for a discussion). This requires analyzing within-student data on both formats, such as we present in Table I. The data from this table support the claim of a hierarchy in performance between the arrow and $i j k$ formats, namely, that answering the $i j k$ format correctly is necessary but not sufficient for answering the arrow format correctly. Put another way, answering the arrow format correctly strongly implies answering the $i j k$ format correctly, but not vice versa. For example, in Table I for the arrow format first, 98\% $(59 / 60)$ of the students answering the arrow format correctly also answered the $i j k$ format correctly. Furthermore (and logically consistent with the hierarchical claim), 91\% (10/11) of students answering the $i j k$ format incorrectly also answered the arrow format incorrectly. Note that the converse is not true, namely, that only $59 \%$ of students answering the $i j k$ format correctly also answered the arrow format correctly, and most (73\%) of the students who answered the arrow format incorrectly nonetheless answered the $i j k$ format correctly. The same pattern, though not as pronounced, is shown in Table I where the students were primed with the $i j k$ format first. Note that in Table I the grid and angle formats were reported together as arrow format. We found that there was no significant difference in the cross tabulations when the results of the two arrow formats were separated.

TABLE I. Within-student cross tabulations of performance on the physics context vector addition questions (Fig. 6) in the arrow format (angle or grid) and the $i j k$ format. Cell values represent counts.

\begin{tabular}{cccc}
\hline \hline \multicolumn{3}{c}{ Arrow format first } \\
\hline \multirow{3}{*}{$i j k$ format } & & \multicolumn{2}{c}{ Arrow format } \\
& Incorrect & 10 & Correct \\
& Correct & 41 & 1 \\
& & & 59
\end{tabular}

ijk format first

\begin{tabular}{lccc} 
& & Incorrect & Correct \\
$i j k$ format & Incorrect & 10 & 5 \\
& Correct & 27 & 66 \\
\hline \hline
\end{tabular}


In short, if a student gets the $i j k$ format wrong, then they will almost certainly get the arrow format wrong. Further, if the student gets the arrow format right, then these results indicate that this student must have already mastered the $i j k$ format, since in this case the $i j k$ format was also answered correctly. This hierarchy supports the idea that learning or understanding the $i j k$ format may be helpful in answering the arrow format correctly. This raises the curious question about whether understanding the $i j k$ format is necessary. At some level this is obviously not true: many algebra-based courses do not use the $i j k$ format; thus, there are certainly students who have never seen the $i j k$ format but who can correctly answer arrow-format questions. However, the results in this paper may suggest that many students do not spontaneously compute in the arrow format correctly, and familiarity with some formal, nonobvious solution path may be necessary, such as the explicit decomposition of the vector into components and subsequent familiar math operations such as subtraction of numbers, which the $i j k$ format does well.

\section{STUDENTS' PHYSICAL SENSE MAKING: ARROW VERSUS $i j k$ FORMAT}

While the above results demonstrate that in many cases students can clearly add and subtract vectors much better in the $i j k$ format, even in some physical contexts (including correct justifications), to what extent does either the arrow or the $i j k$ format facilitate and assess a deeper physical understanding of the physics concepts and processes represented by vector operations? This question is motivated by the notion that the $i j k$ format may prompt the student to perform a mathematical "plug-and-chug" algorithm effectively devoid of instructionally desired deeper physical-spatial meaning. While the results stated earlier for the 2D physical context indicate that at least some students are reasoning physically in the $i j k$ format, we address this question more explicitly in the next two sections.

\section{A. Prompting calculations versus qualitative reasoning}

Given that decades of education research has shown that student responses can be sensitive to context, it is reasonable to expect that the arrow and $i j k$ formats would cue different solution paths. Such differences were evident in the previous sections with the differences in answers and ultimately scores between the two formats. However, those examples highlighted various kinds of incorrect solution paths that can be cued by the context. In this section, we provide an example in which the different formats can cue different but correct paths, including physically intuitive solutions.

As shown by a number of researchers (see, e.g., Refs. $[10,11])$, relatively simple problems can tend to invoke more intuitive solutions. In this spirit, we administered a simpler version of the multiple-force problem used in the previous section by reducing the number of forces in the problem (Fig. 11). The question was presented in either the arrow or $i j k$ format to 103 students, randomly assigned to one of the formats.

Student scores were fairly high in both formats, with the score in the $i j k$ format (88\%) marginally higher than the arrow format score $(78 \%)[t(101)=1.37, p=0.17$, $d=0.27]$.

Further, the solution paths were analyzed and categorized, and the results are presented in Table II, and examples of response types are provided in Fig. 12. The results indicate that the solution paths between the conditions were significantly different $\left[\chi^{2}(5)=36.0\right.$, $p<0.0001]$. In particular, most of the students (55\%) in the $i j k$-format condition solved the problem via a numerical calculation by explicitly adding the components and interpreting the result, whereas only $4 \%$ of the students in the arrow condition used this method. In contrast, most of the students in the arrow-format condition $(60 \%)$ solved the problem by making a valid physical and spatial qualitative argument, whereas only $19 \%$ of students in the $i j k$ format used this method. The dominant correct qualitative argument made was that the horizontal components cancel in each case but the vertical components do not; thus, the sums are different in each case. Another, similar but less common correct argument involved a discussion of the relative angles of the two cases, and the resulting relative vertical components.

In summary, most students could correctly solve this relatively simple problem in either the arrow or $i j k$ format, but students in the $i j k$-format condition tended to solve it with a simple, numerical calculation while students in the arrow condition tended to provide physical, qualitative arguments. Therefore, for the simple problem studied in this section (Fig. 11), the arrow format appears to prompt students to demonstrate their physical-spatial understanding of the problem significantly more than for the $i j k$ format.

\section{B. Are high ijk-format scores a false positive?}

One possible concern is that superior performance on vector operations in the $i j k$ format may not accurately reflect the students' understanding of how the vector operation relates to a relevant physical situation. Put in the colloquial of an instructor, a student might get the $i j k$ format question right, but not "really understand" what they are doing or why they are doing it.

One argument against this would be the results from the previous section, in which it was demonstrated that about $80 \%$ of the students could answer correctly in either format, and that they only tended to explicitly display their physical understanding when prompted with the arrow format; thus, the correct answers in the $i j k$ format were not false 


\section{Arrow-grid format}

Shown below are force diagrams for two different objects, A and B.
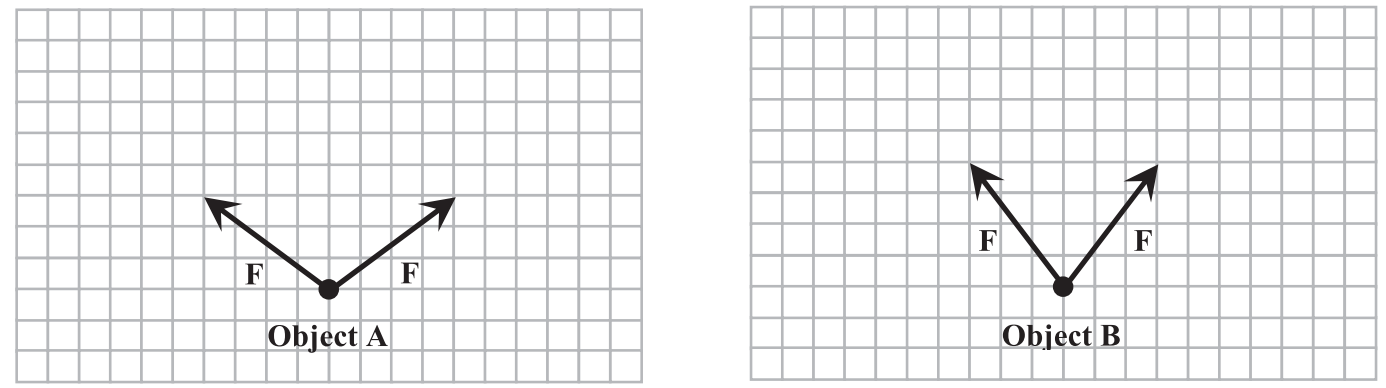

Is the magnitude of the net force on object A greater than, less than, or equal to the magnitude of the net force on object B? Explain or show how you determined your answer.

\section{ijk format}

Listed below are the forces exerted on two different objects, A and B

$$
\begin{aligned}
& \text { Forces on object } \mathrm{C} \\
& \text { (in units of Newtons): } \\
& \text { Forces on object D } \\
& \text { (in units of Newtons): } \\
& \vec{F}_{C 1}=-4 \hat{\imath}+3 \hat{\jmath} \quad \vec{F}_{C 2}=+4 \hat{\imath}+3 \hat{\jmath} \\
& \vec{F}_{D 1}=-3 \hat{\imath}+4 \hat{\jmath} \quad \vec{F}_{D 2}=+3 \hat{\imath}+4 \hat{\jmath}
\end{aligned}
$$

Is the magnitude of the net force on object $\mathrm{C}$ greater than, less than, or equal to the magnitude of the net force on object D? Explain or show how you determined your answer.

FIG. 11. Examples of two-dimensional simplified physics context questions in arrow and $i j k$ format.

positives, the student merely did not display their full physical understanding. However, those results were from a fairly easy problem in which common physical intuition could play a positive role. Further, there was no significant difference in scores between the two conditions. What about for problems in which students score significantly higher in the $i j k$ format than in the arrow format? Is such a high score a kind of false positive, in that students can perform an algorithmic calculation and get the correct answer, but do not have a deeper physical understanding of the problem?

To test this possibility, we administered a physics context question involving momentum impulse which included a vector subtraction calculation, followed by a question asking the student to explain whether the results they obtained made physical sense (Fig. 13). Students
$(N=105)$ were randomly assigned to either the arrowor $i j k$-format question condition.

Performance on the vector calculation was as expected: students scored higher in the $i j k$ format $(77 \%)$ compared to the arrow format $(58 \%)[t(103)=2.04, p=0.04$, $d=0.4]$.

The results on the physical explanation of the calculation were somewhat similar. After analyzing the responses and trying a variety of categorizations, we chose a straightforward binary categorization that would correlate with a correctness score for the response: (1) responses that provided at least some significant and physically correct insight into the solution verses (2) responses that displayed

\begin{tabular}{|c|c|c|c|c|c|c|}
\hline & $\begin{array}{l}\text { Correct, numeric } \\
\text { calculation }\end{array}$ & $\begin{array}{l}\text { Correct, geometric } \\
\text { method }\end{array}$ & $\begin{array}{l}\text { Correct, qualitative } \\
\text { component } \\
\text { cancelling }\end{array}$ & $\begin{array}{l}\text { Correct, qualitative } \\
\text { angle reason }\end{array}$ & $\begin{array}{l}\text { Incorrect, } \\
\text { magnitude reason }\end{array}$ & $\begin{array}{l}\text { Incorrect, } \\
\text { miscellaneous }\end{array}$ \\
\hline$i j k$ format & 55 & 15 & 17 & 2 & 9 & 2 \\
\hline Arrow format & 4 & 17 & 48 & 12 & 14 & 5 \\
\hline
\end{tabular}
incorrect or little to no coherent physical insight. Examples are provided in Table III. Responses in the first category explicitly acknowledged that the momentum changed in a

TABLE II. Percentage of students using various solution methods for the physical context vector addition question (Fig. 11) for both the arrow and $i j k$ formats. 


\begin{tabular}{|c|c|}
\hline $\begin{array}{c}\begin{array}{c}\text { Correct, geometric reasoning } \\
\text { (ijk-format condition) }\end{array} \\
\text { thet force on } \mathrm{C} \text { is less than }\end{array}$ & $\begin{array}{l}\text { The net force an } \mathrm{C} \text { is less than } \\
\text { Net force on object } \mathrm{D} \text {. I solved } \\
\text { this by puthing forces "arrowto } \\
\text { tail" of solving for net force. }\end{array}$ \\
\hline  & $\begin{array}{l}\begin{array}{l}\text { Correct, qualitative canceling of components } \\
\text { (arrow-format condition) }\end{array} \\
\text { "Objects D \& C have horizontal forces that cancel, to } \\
\text { determine the greater net force we must look at the } \\
\text { vertical components of the forces. Each force on C has } 3 \\
\text { units upwards, each force on object D has } 4 \text { units } \\
\text { upwards. Object D then has the greater net force." }\end{array}$ \\
\hline $\begin{array}{l}\begin{array}{l}\text { Correct, qualitative angle reason } \\
\text { (arrow format condition) }\end{array} \\
\text { "Object B has a smaller angle between forces, creating a } \\
\text { greater vertical force. Therefore, Net force on B > Net } \\
\text { force on A." }\end{array}$ & $\begin{array}{l}\begin{array}{l}\text { Incorrect, magnitude reason } \\
\text { (arrow-format condition) }\end{array} \\
\text { "F=ma. The Net force on object } C \text { is equal to that of } \\
\text { object D as long as all of the forces are equal." }\end{array}$ \\
\hline
\end{tabular}

FIG. 12. Assortment of example student solutions to simple two-dimensional physics context questions (Fig. 11) in both arrow and $i j k$ formats. Response rates are in Table II.

specific direction (e.g., negative $x$ direction, or left) and this change in momentum required an average net force in that direction. A minority of students $(11 \%$ in the arrow format and $21 \%$ in the $i j k$ format) also correctly stated that the momentum in the $y$ direction does not change; thus, the average force in the $y$ direction was zero. We were somewhat generous in our interpretations of the student responses, and responses in the second category indeed had very little if any redeeming quality.

Overall, the fraction of partially or fully correct responses to the physical explanation question were about the same in each condition, with $60 \%$ in the $i j k$-format condition and $49 \%$ in the arrow-format condition $[\chi(1)=1.18, p=0.28, d=0.2]$. One might argue that the students in the $i j k$-format condition would tend to score better in explanation than those in the arrow-format condition simply because they calculated the correct answer more frequently. Indeed, the correlation between the correct calculation and the correct physical interpretation was $\varphi=0.31$. Yet there is some indication that the $i j k$ format has some additional benefit: of the students answering the calculation correctly, $58 \%$ of the students in the arrow format and $70 \%$ in the $i j k$ format also answered the physical explanation correctly. Though this difference is not statistically significant $[\chi(1)=1.09, \quad p=0.3$, $d=0.25]$, the effect size is approaching an important magnitude, and the sample size is smaller since we are selecting only those $\sim 65 \%$ of students who answered correctly.

In summary, at least for this example, most students calculating answers correctly in the $i j k$ format also provided physical explanations of their answers at least as often if not more often than students answering in the arrow format. Thus, in this case there was no evidence that the high success rates for questions in the $i j k$ format were false positives, and if anything, there was an indication that the $i j k$ format was providing a context in which the student was able to gain (or demonstrate) a better physical-spatial understanding of the problem compared to the arrow format. 


\section{Arrow-grid format}

From Newton's Laws we know that the average net force on an object $\overrightarrow{\boldsymbol{F}}_{\text {ave }}$ is pointed in the direction of the change in momentum $\Delta \vec{p}$ :

$$
\vec{F}_{\text {ave }}=\frac{\Delta \vec{p}}{\Lambda t}=\frac{\vec{p}_{f}-\vec{p}_{i}}{\Lambda t}
$$

Consider an object with initial momentum $\vec{p}_{i}$ and final momentum $\vec{p}_{f}$, as shown below in standard SI units for momentum: one square is $1 \mathrm{~kg} \mathrm{~m} / \mathrm{s}$. The time interval is $\Delta t=t_{f}-t_{i}=1 \mathrm{~s}$.
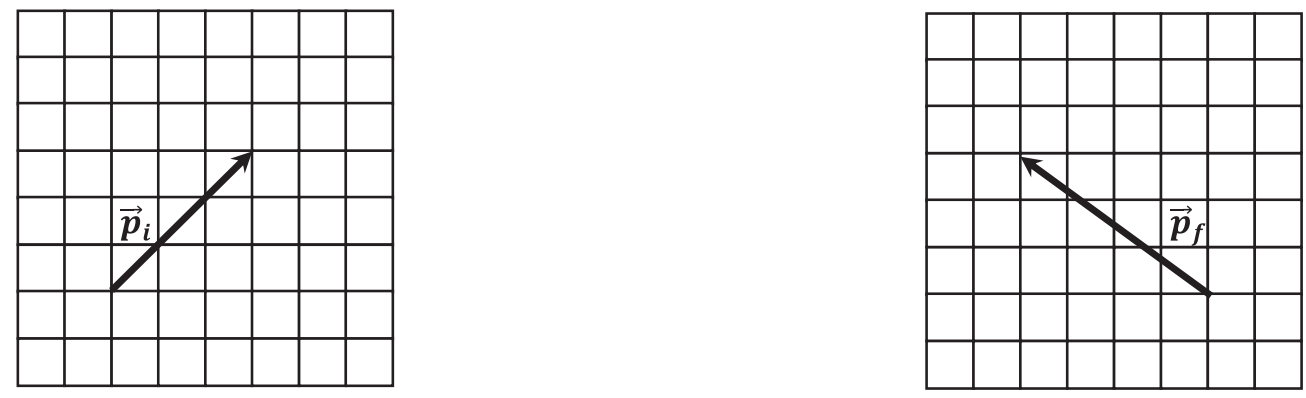

What is the average force $\vec{F}_{\text {ave }}$ on the object during the time $\Delta t$ ? Draw your answer on the grid to the right (which is in standard SI units for force: one square is $1 \mathrm{~N})$ :

Does the direction of $\vec{F}_{\text {ave }}$ make sense physically? Briefly explain.

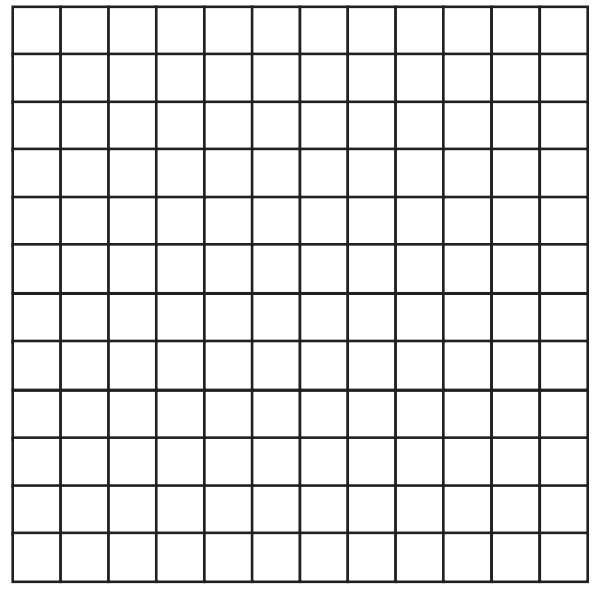

\section{$i j k$ format}

From Newton's Laws we know that the average net force on an object $\overrightarrow{\boldsymbol{F}}_{\text {ave }}$ is pointed in the direction of the change in momentum $\Delta \vec{p}$ :

$$
\vec{F}_{\text {ave }}=\frac{\Delta \vec{p}}{\Lambda t}=\frac{\vec{p}_{f}-\vec{p}_{i}}{\Lambda t}
$$

Consider an object with initial momentum $\vec{p}_{i}$ and final momentum $\vec{p}_{f}$, as shown below in standard SI units for momentum: one square is $1 \mathrm{~kg} \mathrm{~m} / \mathrm{s}$. The time interval is $\Delta t=t_{f}-t_{i}=1 \mathrm{~s}$.

$$
\vec{p}_{i}=3 \hat{\imath}+3 \hat{\jmath} \quad \vec{p}_{i}=-4 \hat{\imath}+3 \hat{\jmath}
$$

What is the average force $\vec{F}_{\text {ave }}$ on the object during the time $\Delta t$ ? Write your answer below (assume the answer is in standard SI units for force $(\mathrm{N}))$ :

Does the direction of $\vec{F}_{\text {ave }}$ make sense physically? Briefly explain.

FIG. 13. Change in momentum physics context questions in arrow and $i j k$ format. 
TABLE III. Examples of responses counted as at least partially correct to momentum-impulse question (Fig. 13) explaining why the numerical response obtained makes physical sense.

\begin{tabular}{|c|c|}
\hline Condition & Student response \\
\hline Arrow format & Yes because it changes the direction to the left and up from the right and up. \\
\hline Arrow format & $\begin{array}{l}\text { Yes it does. A force acting to the left changes the direction of velocity from a general Northwest Direction to a } \\
\text { Northeast direction. But no vertical force acts. }\end{array}$ \\
\hline$i j k$ format & $\begin{array}{l}\text { Yes the momentum in the y direction remains the same therefore there is no component of force in that direction. } \\
\text { The momentum change from } 3 \mathrm{~kg}-\mathrm{m} / \mathrm{s} \text { to }-4 \mathrm{~kg}-\mathrm{m} / \mathrm{s} \text { in the } x \text { direction. Therefore the force was applied in the } \\
\qquad-x \text { direction }\end{array}$ \\
\hline$i j k$ format & Yes. It is initially going right and quickly $(1 \mathrm{~s})$ changes going faster to the left. The force would have to be to the left. \\
\hline
\end{tabular}

\section{SUMMARY AND GENERAL DISCUSSION}

This study presents two general findings. First, this study systematically studies and carefully characterizes specific student difficulties with adding and subtracting vectors in the arrow format. We replicated past general findings of student difficulties with these tasks in the arrow format and extended these findings to uncover new difficulties and give more detailed descriptions of a number of difficulties with vector subtraction in the arrow format. For example, we found that in one dimension, many students tended to correctly subtract aligned arrows but incorrectly subtracted opposing arrows (by essentially adding them); however, in two dimensions, qualitatively different mistakes were elicited, and many students would even incorrectly subtract arrows that were pointed in roughly the same direction in addition to making mistakes for arrows with at least one opposing dimension. This is a clear indication that if instruction and practice only include one-dimensional tasks, students will not be able to practice and overcome important difficulties found in two-dimensional tasks that are not simple extensions of multiple one-dimensional tasks, especially in the arrow format.

Second, there are important differences in student performance between equivalent arrow- versus $i j k$-format tasks. Most notably, student difficulties with adding and subtracting vectors in the arrow format were brought into sharp relief when compared to performance on these questions in the $i j k$ format, where the percentage of correct solutions is usually dramatically better, often at $90 \%$ for both high and low grade students. This superior $i j k$-format performance was found in one- and two-dimensional tasks and for both generic mathematical and physical contexts.

On closer inspection of student responses, we found that the arrow and $i j k$ formats can prompt students to significantly different solution paths. Perhaps as expected, the arrow format tends to prompt more spatial-intuitive reasoning and the $i j k$ format prompts more numeric-algebraic calculations. However, students are also able to make good physical-spatial arguments in the $i j k$ format, especially when prompted to do so. Therefore, at least for the examples we studied, the relative high accuracy performance in the $i j k$ format does not appear to be an artifact of thoughtless "plug-and-chug" application of a mathematical algorithm, but simply another productive (perhaps more productive) solution path, one that is less wrought with the prior conceptions and habits that accompany tasks in an arrow format.

On the other hand, while the $i j k$ format may be more useful for learning important vector concepts and for accurate calculation, in some ways the arrow format may serve better for assessing understanding of and performance on vector tasks involving sums and differences. Specifically, we found empirically that the arrow format is hierarchically at a higher level than the $i j k$ format: answering the arrow format correctly implies being able to answer the $i j k$ format correctly. That is to say that these results suggest that a complete understanding of the arrow format requires an understanding of numerical decomposition of the full arrow vector into vector components as represented, for example, by the $i j k$ format. This need for the mindset of decomposing the arrow format into components was highlighted by the demonstration that priming students with $i j k$-format questions helps them to solve arrow-format questions. Finally, using the arrow format is an important instructional goal itself, and since we have found that the arrow format has its own issues, assessing in the arrow format is indispensable.

\section{A. Implications for instruction}

While this study did not include instructional interventions beyond what typically occurs in traditional physics classrooms, our results do suggest instructional methods to improve instruction, described below. Of course, these suggestions warrant explicit studies to determine their effectiveness.

\section{Concurrently use the arrow- and ijk-format representations}

Noncalculus-based physics courses at the high school and university level often do not introduce the $i j k$-format representation. Our results suggest that introducing both representations may have the advantages of the ease of calculation via the $i j k$ format while also benefiting from and achieving the instructional goal of student practice with 
and understanding of the intuitive and physical-spatially descriptive arrow format. It is possible that the $i j k$ format may prove to be too formal and unfamiliar for younger or less mathematically prepared students, and some simpler notation may need to be used, but our findings suggest that this (or perhaps similar) notation is more naturally and hierarchically fundamental for applying vector sum and difference operations. Note also that the arrow format can be in the grid or angle format, so this is another aspect to practice. Furthermore, there are likely added benefits to having students practice and reason with multiple representations (cf. Refs. [12,13]). When translating between representations, one of the benefits may be the natural need to explicitly discuss vector decomposition, which is a very useful method for many vector calculations.

We also note another possible instructional intervention not mentioned here, namely, practicing and learning vectors via computer coding, such as was advocated by Chabay and Sherwood [14]. Representing a vector in a computer code format is yet a third representation that could be useful for helping students to gain yet more insights into the nature of vectors and also help to overcome the disadvantages of the arrow representation.

\section{Practice with a variety of relative orientations between vectors}

Our results indicate that student thinking and responses can strongly depend on the relative orientation of the vectors in a task. Therefore, students should practice with a variety of orientations covering all categories of possibilities, with special attention paid to difficult orientations. For example, students have different kinds of difficulties with subtraction in 1D for opposing vectors, subtraction in 2D when components in one dimension are opposed, and when components in both dimensions are opposed or in the same direction.

\section{B. Discussion and conclusion}

Perhaps the most cogent point from this study is that the relation between the arrow- and $i j k$-format representations of vectors is analogous to the relation between the graph and equation representations of functions. Arrows and graphs are powerful, intuitive pictorial representations that can help students to quickly gain deeper physical insight into problems, yet they also have significant prior knowledge and experience "baggage" that can cause students to use them in familiar, everyday ways that result in incorrect (undesired) extensions for physics problems. It is well known, for example, that students often interpret graphs as "pictures" [15-17], which is correct in some cases, but not for others. For arrows, students often picture opposing arrows as "competing" and, regardless of the assigned task, will simply tally the result of two opposing "forces." In contrast, equations and $i j k$ format are both symbolic, algebraic expressions that tend to cue arithmetic and algebraic calculations. The task then becomes one of overcoming the incorrect use of the pictorial representations and melding a deeper connection of them with the symbolic ones.

Clearly, in both cases, these pictorial representations are highly useful as a conceptual and semiquantitative representation, a qualitative sense-making tool, and an efficient means for communicating. For vector arrows, one example is the abundant number of textbook examples of free-body force diagrams in the arrow format. Another important example in introductory physics is the derivation of the direction of acceleration for an object moving in a circle. Virtually all introductory physics textbooks derived this via the arrow representation-doing this algebraically would likely be less instructive and intuitive for the students.

Nonetheless, students do have difficulties with these pictorial representations, and this study shows that students are having difficulties with the arrow representation itself, rather than the physics behind it. The evidence is that, for the questions studied here, many more students could answer correctly, including with correct justification, in the $i j k$ format than could answer correctly in the arrow format. This suggests that the $i j k$ format is not only a useful calculational tool, but it may also be a very useful but perhaps underutilized instructional tool for learning concepts and physical phenomena involving vectors.

\section{ACKNOWLEDGMENTS}

This work has been supported in part by the Center for Emergent Materials at The Ohio State University, a NSF MRSEC (Award No. DMR-1420451).
[1] L. Nguyen and D. E. Meltzer, Initial understanding of vector concepts among students in introductory physics courses, Am. J. Phys. 71, 630 (2003).

[2] S. Flores, S. E. Kanim, and C. H. Kautz, Student use of vectors in introductory mechanics, Am. J. Phys. 72, 460 (2004).
[3] P. S. Shaffer and L. C. McDermott, A research-based approach to improving student understanding of the vector nature of kinematical concepts, Am. J. Phys. 73, 921 (2005).

[4] J. Van Deventer and M. C. Wittmann, Comparing student use of mathematical and physical vector representations, AIP Conf. Proc. 951, 208 (2007). 
[5] P. Barniol and G. Zavala, Vector addition: Effect of the context and position of the vectors, AIP Conf. Proc. 1289, 73 (2010).

[6] J. M. Hawkins, J. R. Thompson, and M. C. Wittmann, Students' consistency of graphical vector addition method on 2-D vector addition tasks, AIP Conf. Proc. 1179, 161 (2009).

[7] P. Barniol and G. Zavala, Test of understanding of vectors: A reliable multiple-choice vector concept test, Phys. Rev. ST Phys. Educ. Res. 10, 010121 (2014).

[8] R. D. Knight, Phys. Teach. 33, 74 (1995).

[9] R. Rosenblatt and A. F. Heckler, Systematic study of student understanding of the relationships between the directions of force, velocity, and acceleration in one dimension, Phys. Rev. ST Phys. Educ. Res. 7, 020112 (2011).

[10] K. R. Koedinger and M. J. Nathan, The real story behind story problems: Effects of representations on quantitative reasoning, J. Learn. Sci. 13, 129 (2004).

[11] A. F. Heckler, Some consequences of prompting novice physics students to construct force diagrams, Int. J. Sci. Educ. 32, 1829 (2010).
[12] A. Van Heuvelen, Learning to think like a physicist: A review of research-based instructional strategies, Am. J. Phys. 59, 891 (1991).

[13] How Students Learn: History, Mathematics, and Science in the Classroom, edited by M.S. Donovan and J.D. Bransford (National Academies Press, Washington, DC, 2005).

[14] R. Chabay and B. Sherwood, Computational physics in the introductory calculus-based course, Am. J. Phys. 76, 307 (2008).

[15] L. C. McDermott, M. L. Rosenquist, and E. H. Van Zee, Student difficulties in connecting graphs and physics: Examples from kinematics, Am. J. Phys. 55, 503 (1987).

[16] J. R. Mokros and R. F. Tinker, The impact of microcomputerbased labs on children's ability to interpret graphs, J. Res. Sci. Teach. 24, 369 (1987).

[17] R. J. Beichner, Testing Student Interpretation of Kinematics Graphs, Am. J. Phys. 62, 750 (1994). 